\title{
Spacelike hypersurfaces with functionally bounded mean curvature in Lorentzian warped products and generalized Calabi-Bernstein type problems
}

\author{
Juan A. Aledo ${ }^{a}$, Rafael M. Rubio ${ }^{b}$ and Juan J. Salamanca ${ }^{c}$ \\ ${ }^{a}$ Departamento de Matemáticas, E.S.I. Informática, \\ Universidad de Castilla-La Mancha, 02071 Albacete, Spain, \\ E-mail: juanangel.aledo@uclm.es \\ ${ }^{b},{ }^{c}$ Departamento de Matemáticas, Campus de Rabanales, \\ Universidad de Córdoba, 14071 Córdoba, Spain, \\ E-mails: rmrubio@uco.es, jjsalamanca@uco.es
}

Dedicated to Professor Alfonso Romero on the occasion of his 60th birthday

\begin{abstract}
We study spacelike hypersurfaces with functionally bounded mean curvature in Lorentzian warped products $\bar{M}=I \times{ }_{f} F$, where $F$ is a (non-compact) complete Riemannian manifold whose universal covering is parabolic. In particular, we provide several rigidity results under appropriate mathematical and physical assumptions. As an application, several CalabiBernstein type results are obtained which widely extend the previous ones in this setting.
\end{abstract}

MSC 2010 53C21, 58J05, 53B30.

Keywords: Lorentzian warped products, spacelike hypersurfaces, mean curvature, parabolicity, Calabi-Bernstein problems.

\section{Introduction}

In this work we are interested in providing some rigidity results for spacelike hypersurfaces whose mean curvature is controlled by natural geometrical functions of the ambient spacetime. Recall that a maximal spacelike hypersurface $M$ immersed in an $(n+1)$-dimensional Lorentzian manifold $\bar{M}, \psi: M \longrightarrow \bar{M}$, is a hypersurface whose induced metric via $\psi$ is Riemannian and whose mean curvature $H$ vanishes identically. This terminology comes from the fact that such hypersurfaces 
locally maximize the $n$-volume among all nearby hypersurfaces having the same boundary values (see [13] and [21]). The study of such hypersurfaces has attracted the attention of a great deal of researchers due to several mathematical and physical reasons.

From a physical point of view, these hypersurfaces and more generally those spacelike hypersurfaces with constant mean curvature (CMC), have been used as initial data for solving the Cauchy problem of the Einstein equation. In fact, Lichnerowicz [25] showed that such Cauchy problem reduces to a linear differential system of first-order and to a non-linear second-order elliptic differential equation when a maximal hypersurface is considered as initial data. On the other hand, maximal hypersurfaces can be used to describe the transition between expansive and contractive phases in several relevant models of the universe [12].

From a mathematical point of view, maximal hypersurfaces present interesting Bernstein-type properties. As is well-known, the Calabi-Bernstein theorem states that the only entire solutions to the equation for maximal hypersurfaces in the Lorentz-Minkowski spacetime $\mathbb{L}^{n+1}$ are the spacelike affine hyperplanes. This central result was firstly proved by Calabi [14] for $n \leq 4$ and later extended to arbitrary dimension by Cheng and Yau [18]. Moreover, this result has motivated the study of entire maximal graphs in more general Lorentzian spacetimes. In the particular case when $\bar{M}=I \times{ }_{f} F$ is a Lorentzian warped product (see Section 2), the mean curvature of a graph $\Sigma_{u}=\{(u(p), p): p \in F\}$ is given by the operator

$$
H(u)=\operatorname{div}\left(\frac{D u}{n f(u) \sqrt{f(u)^{2}-|D u|^{2}}}\right)+\frac{f^{\prime}(u)}{n \sqrt{f(u)^{2}-|D u|^{2}}}\left(n+\frac{|D u|^{2}}{f(u)^{2}}\right),
$$

where div and $D$, denote the divergence and the gradient operators, respectively, in the Riemannian manifold $F$. Observe that the case $f \equiv 1$ corresponds to Lorentzian products and the mean curvature operator reduces to

$$
H(u)=\operatorname{div}\left(\frac{D u}{n \sqrt{1-|D u|^{2}}}\right)
$$

Hence, the equation for maximal spacelike graphs becomes $H(u)=0$ jointly with a constraint condition to assure that the graph is spacelike. For $n=2$ and $f \equiv 1$ Albujer and Alías $[1$, Cor. 8] proved a uniqueness result for entire solutions in the the maximal case, when the Gaussian curvature of $F$ is non-negative and positive at some point (see also [2], [20]).

In the particular case when $F=\mathbb{R}^{2}$, Latorre and Romero [23] provided a uniqueness type result for the equation $H(u)=0$ when the warping function $f$ is non locally constant and satisfies that $f^{\prime \prime} \leq 0$. The same result was obtained by Romero and Rubio [31] by means of an upper bound for the integral, on a geodesic disc, of the squared length of the gradient of a distinguished function.

More general extensions can be found in more recent papers [15], [16] when $F$ is a (non-compact) complete Riemannian surface under appropriate conditions for its Gaussian curvature and certain assumptions on the warping function as convexity of its logarithm. For arbitrary dimension, and provided that the (non-compact) complete Riemannian manifold $F$ has a parabolic universal Riemannian covering, several uniqueness and non-existence results for the equation $H(u)=0$ have been recently obtained in [32] and [34]. On the other hand, Li and Salavessa generalized a Bernstein-type result due to Albujer and Alías [2] for maximal surfaces in a 3-dimensional Lorentzian product, to higher dimension and codimension [24]. It worths pointing out that in 
the simpler case when $F$ is a (without boundary) compact manifold, the equation $H(u)=$ cte has been widely studied in various papers [7], [8], [17]. In fact, if $F$ is compact then every closed entire spacelike graph is complete. Nevertheless, in general the induced metric on a closed entire spacelike graph in a complete Lorentzian manifold could be geodesically non-complete (see, for instance, [6]) in contrast to what happens in Riemannian ambients.

A natural generalization of this kind of problems consists in considering differential inequations involving the operator $H(u)$ to obtain several rigidity results for spacelike hypersurfaces. In a previous paper [30], Romero and Rubio obtained rigidity results for spacelike complete surfaces immersed in a warped product $I \times \mathbb{R}^{2}$ such that its mean curvature at every point does not exceed to the mean curvature of the spacelike slice through this point, under the additional assumption $f^{\prime \prime} \leq 0$. They also solved the corresponding Calabi-Bernstein type problem. A more general approach to this problem on Riemannian surfaces with finite total curvature is given in [33]. In [35], Wang and Liu studied the parametric version of a similar problem for arbitrary dimension making use of a technique provided by Yau [36]. A non-parametric approach for this type of problem is given in [10] by Aquino and de Lima, when the mean curvature is constant. Also in the case of constant mean non-zero curvature Albujer, Camargo and de Lima studied a differential inequation in a Lorentzian warped product whose fiber has constant sectional curvature [3]. On the other hand, Colares and de Lima [19], applying a maximum principle at the infinity due to Yau, obtained a uniqueness result for complete hypersurfaces immersed in a semi-Riemannian warped product whose warping function has convex logarithm, under the assumption that the hypersurface is timelike bounded and its mean curvature satisfies a natural inequality involving the mean curvature of the slices which determine the region where the hypersurface is contained.

The aim of this paper is to provide uniqueness and non-existence results to several generalized Calabi-Bernstein type problems on (non-compact) complete parabolic Riemannian manifolds. More specifically, we deal with differential inequalities $H(u)^{2} \leq \beta(u)$ which widely extend the maximal case, where the real functions $\beta$ will arise from natural geometrical situations involving the warping function $f$. Our first approach to these problems will be geometric, so providing some parametric type rigidity results (see Section 3). Next, making use of these results, we study several Calabi-Bernstein type problems (see Section 4) which widely extend the previous ones in this setting.

\section{Preliminaries}

Let $\left(F, g_{F}\right)$ be an $n$-dimensional $(n \geq 2)$ connected Riemannian manifold and $I \subseteq \mathbb{R}$ an open interval in $\mathbb{R}$ endowed with the metric $-d t^{2}$. The warped product $\bar{M}=I \times_{f} F$ endowed with the Lorentzian metric

$$
\bar{g}=-\pi_{I}^{*}\left(d t^{2}\right)+f\left(\pi_{I}\right)^{2} \pi_{F}^{*}\left(g_{F}\right)
$$

where $f>0$ is a smooth function on $I$, and $\pi_{I}$ and $\pi_{F}$ denote the projections onto $I$ and $F$ respectively, is said to be a Lorentzian warped product or, in physical contexts, a Generalized Robertson-Walker $(G R W)$ spacetime with fiber $\left(F, g_{F}\right)$, base $\left(I,-d t^{2}\right)$ and warping function $f$ (see $[7])$.

These Lorentzian warped products widely extend the classical Robertson-Walker spacetimes, whose fiber have constant sectional curvature. Recall that the family of Robertson-Walker space- 
times includes the usual big-bang cosmological models. Contrary to these spacetimes, our ambient spacetimes are not necessarily spatially-homogeneous. Note that to be spatially-homogeneous, which is reasonable as a first approximation of the large scale structure of the universe, could not be appropriate when we consider a more accurate scale.

The coordinate vector field $\partial_{t}:=\partial / \partial t$ globally defined on $\bar{M}$ is (unitary) timelike, and so $\bar{M}$ is time-orientable. We will also consider on $\bar{M}$ the conformal closed timelike vector field $K:=$ $f\left(\pi_{I}\right) \partial_{t}$. From the relationship between the Levi-Civita connections of $\bar{M}$ and those of the base and the fiber [27, Cor. 7.35], it follows that

$$
\bar{\nabla}_{X} K=f^{\prime}\left(\pi_{I}\right) X
$$

for any $X \in \mathfrak{X}(\bar{M})$, where $\bar{\nabla}$ is the Levi-Civita connection of the Lorentzian metric (1).

A smooth immersion $\psi: M^{n} \longrightarrow \bar{M}$ of an $n$-dimensional (connected) manifold $M$ is said to be a spacelike hypersurface if the induced metric $g$ via $\psi$ is a Riemannian metric on $M$.

Since $\bar{M}$ is time-orientable we can take, for each spacelike hypersurface $M$ in $\bar{M}$, a unique unitary timelike vector field $N \in \mathfrak{X}^{\perp}(M)$ globally defined on $M$ with the same time-orientation as $\partial_{t}$, i.e. such that $\bar{g}\left(N, \partial_{t}\right)<0$. From the wrong-way Cauchy-Schwarz inequality (see [27, Prop. 5.30], for instance), we have $\bar{g}\left(N, \partial_{t}\right) \leq-1$, and the equality holds at a point $p \in M$ if and only if $N=\partial_{t}$ at $p$. The hyperbolic angle $\varphi$, at any point of $M$, between the unit timelike vectors $N$ and $\partial_{t}$, is given by $\bar{g}\left(N, \partial_{t}\right)=-\cosh \varphi$.

We will denote by $A$ and $H:=-(1 / n) \operatorname{tr}(A)$ the shape operator and the mean curvature function associated to $N$. A spacelike hypersurface with $H=0$ is called a maximal hypersurface. The reason for this terminology is that the mean curvature is zero if and only if the spacelike hypersurface is a local maximum of the $n$-dimensional area functional for compactly supported normal variations.

In any Lorentzian warped product $\bar{M}$ there is a remarkable family of spacelike hypersurfaces, namely its spacelike slices $\left\{t_{0}\right\} \times F, t_{0} \in I$. The spacelike slices constitute for each value $t_{0}$ the restspace of the distinguished observers in $\partial_{t}$. A spacelike hypersurface in $\bar{M}$ is a (piece of) spacelike slice if and only if the function $\tau:=\pi_{I} \circ \psi$ is constant. Furthermore, a spacelike hypersurface in $\bar{M}$ is a (piece of) spacelike slice if and only if the hyperbolic angle $\varphi$ vanishes identically.

The shape operator and the mean curvature of the spacelike slice $t=t_{0}$ are, respectively, $A=$ $-f^{\prime}\left(t_{0}\right) / f\left(t_{0}\right) I$, where $I$ denotes the identity transformation, and the constant $H=f^{\prime}\left(t_{0}\right) / f\left(t_{0}\right)$. Thus, a spacelike slice $t=t_{0}$ is maximal if and only if $f^{\prime}\left(t_{0}\right)=0$ (and hence, totally geodesic).

If we put $\partial_{t}^{T}=\partial_{t}+\bar{g}\left(\partial_{t}, N\right) N$ the tangential part of $\partial_{t}$ and $N^{F}=N+\bar{g}\left(N, \partial_{t}\right) \partial_{t}$ the projection of $N$ onto the fiber, it follows by using that both $N$ and $\partial_{t}$ are unitary timelike vectors that

$$
\left|\partial_{t}^{T}\right|^{2}=\left|N^{F}\right|^{2}=\sinh ^{2} \varphi
$$

Hence, a spacelike hypersurface $M$ in $\bar{M}$ is a (piece of) spacelike slice if and only if $\left|\partial_{t}^{T}\right|^{2}=\left|N^{F}\right|^{2}$ vanishes identically on $M$.

We will say that the spacelike hypersurface is contained in a slab, if it is contained between two slices. Analogously, we will say that the hypersurface is contained in an open slab if it is contained in the unbounded region determined for a slice, i.e. if there exists $t_{0} \in I$ such that $\tau>t_{0}$ or $\tau<t_{0}$ on $M$. 


\subsection{Parabolicity in Lorentzian warped products}

A Lorentzian warped product $\bar{M}=I \times_{f} F$ is said to be spatially parabolic [32] if its fiber is parabolic; i.e. it is a non-compact complete Riemannian manifold such that the only superharmonic functions on it which are bounded from below are the constants. Analogously, a Lorentzian warped product is said to be spatially parabolic covered if its universal Lorentzian covering is spatially parabolic. Observe that the universal Lorentzian covering of $I \times_{f} F$ is $I \times_{f} \widetilde{F}$, where $\widetilde{F}$ is the universal Riemannian covering of the fiber $F$. In particular, every spatially parabolic Lorentzian warped product is spatially parabolic covered, and both notions agree when the fiber is simply-connected.

The spatially parabolic GRW spacetimes are a natural counterpart of the well-known family of spatially closed GRW spacetimes, i.e. those whose fiber is compact. In fact, the parabolicity of the fiber implies a nice control of the geometry of the physical space. For instance, when the volume (or the area) growth of the geodesic balls has a certain behavior, such parabolicity is attained [22]. The assumption of parabolicity can be also suitable to describe physical spaces with a certain symmetry. Among them, it worths mentioning the quasi-spherical case (see [11]). From a physical point of view, an interesting fact occurs when the fiber is parabolic: as galaxies can be understood as molecules, if a sonde is sent to the space its motion may be approached by a Brownian motion. In fact, the distribution of galaxies and their velocities are not completely known. Thus, parabolicity may favor that the sonde can be observed in any region, since the Brownian motion is recurrent in any parabolic Riemannian manifold (see [22]).

Lorentzian warped products which admit a complete parabolic spacelike hypersurface have been studied in [32], where the following result is proved:

Let $M$ be a complete spacelike hypersurface in a spatially parabolic covered Lorentzian warped product $\bar{M}=I \times{ }_{f} F$. If the hyperbolic angle of $M$ is bounded and the restriction $f(\tau)$ on $M$ of the warping function $f$ satisfies:

i) $\sup f(\tau)<\infty$, and

ii) $\inf f(\tau)>0$,

then $M$ is parabolic.

This result will be used in Section 3. Another way to get parabolicity on $M$ is through a pointwise conformal change of metric (see [34, Theorem 4]):

Let $M$ be a complete spacelike hypersurface in a spatially parabolic covered Lorentzian warped product $\bar{M}=I \times{ }_{f} F$. If $\sup f(\tau)<\infty$ and the hyperbolic angle of $M$ is bounded, then $M$, endowed with the conformal metric $\widehat{g}=\frac{1}{f(\tau)^{2}} g$, is parabolic.

In particular, when $n=2$ the superharmonic functions on $(M, g)$ and $(M, \widehat{g})$ coincide, and so, as $(M, g)$ is complete, parabolicity on $(M, g)$ follows from parabolicity on $(M, \widehat{g})$. Regarding the general case of arbitrary dimension $n \geq 2$, the following result is proved in [34, Proposition 7])

Let $\bar{M}=I \times_{f} F$ be a Lorentzian warped product which admits a simply-connected spacelike hypersurface $M$ such that $\sup f(\tau)<\infty$, the hyperbolic angle of $M$ is bounded, 
and the conformal metric $\frac{1}{f(\tau)^{2}} \mathrm{~g}$ is parabolic on $M$. Then $\left(\widetilde{F}, g_{\widetilde{F}}\right)$ is also parabolic and, in particular, $\bar{M}$ is spatially parabolic.

\section{Parametric type results}

Let $\psi: M \rightarrow \bar{M}$ be a spacelike hypersurface in a Lorentzian warped product $\bar{M}=I \times_{f} F$. It is easy to check that the gradient of $\tau=\pi_{I} \circ \psi$ on $M$ is given by

$$
\nabla \tau=-\partial_{t}^{T}
$$

and its Laplacian by

$$
\Delta \tau=-\frac{f^{\prime}(\tau)}{f(\tau)}\left\{n+|\nabla \tau|^{2}\right\}-n H \bar{g}\left(N, \partial_{t}\right)
$$

Let us take $G: I \longrightarrow \mathbb{R}$ such that $G^{\prime}=f$. Using (4) we have that the gradient of $G(\tau)$ on $M$ is given by

$$
\nabla G(\tau)=G^{\prime}(\tau) \nabla \tau=-f(\tau) \partial_{t}^{T}=-K^{T},
$$

where $K^{T}=K+\bar{g}(K, N) N$ is the tangential component of $K$ along $\psi$, and so its Laplacian on $M$ (see $[7$, Eq. 6])) yields

$$
\Delta G(\tau)=\operatorname{div}(\nabla G(\tau))=-n f^{\prime}(\tau)-n H \bar{g}(K, N) .
$$

Analogously, a direct computation from (2) gives

$$
\nabla \bar{g}(K, N)=-A K^{T},
$$

where we have also used (4), and so the Laplacian of $\bar{g}(K, N)$ on $M$ becomes (see [7, Eq. 8])

$$
\Delta \bar{g}(K, N)=\operatorname{div}(\nabla \bar{g}(K, N))=\overline{\operatorname{Ric}}\left(K^{T}, N\right)+n \bar{g}(\nabla H, K)+n f^{\prime}(\tau) H+\bar{g}(K, N) \operatorname{tr}\left(A^{2}\right) .
$$

Now, let us consider on $M$ the metric $\widehat{g}=\frac{1}{f(\tau)^{2}} g$, which is pointwise conformal to the induced metric $g$. If we denote by $\widehat{\Delta}$ the Laplacian with respect to the metric $\widehat{g}$, then from (5) we get

$$
\widehat{\Delta} \tau=-f(\tau) f^{\prime}(\tau)\left\{n+(n-1)|\nabla \tau|^{2}\right\}-n H f(\tau)^{2} \bar{g}\left(N, \partial_{t}\right)
$$

and

$$
\begin{aligned}
\widehat{\Delta} f(\tau)= & -n f(\tau) f^{\prime}(\tau)^{2}-n H f^{\prime}(\tau) f(\tau)^{2} \bar{g}\left(N, \partial_{t}\right) \\
& +f(\tau)^{3}\left\{(\log f)^{\prime \prime}(\tau)-(n-2) \frac{f^{\prime}(\tau)^{2}}{f(\tau)^{2}}\right\}|\nabla \tau|^{2} .
\end{aligned}
$$

In what follows, in order to make easier the statements of the results, we will adopt the following convention: the function $f: I \longrightarrow(0,+\infty)$ will be said to be globally constant if $I=\mathbb{R}$ and $f$ is constant. Recall that a Lorentzian warped product $M=I \times{ }_{f} F$ is said to be static or simply a 
Lorentzian product if the warping function $f$ is constant on its domain $I$ (non-necessarily $I=\mathbb{R}$ ). In particular, if the warping function $f$ is globally constant then $\bar{M}=I \times_{f} F$ is a Lorentzian product, but the converse is not true since $I$ could be different from $\mathbb{R}$.

Next we characterize the spacelike slices of a spatially parabolic covered Lorentzian warped product whose warping function satisfies that $(\log f)^{\prime \prime} \leq \alpha(\log f)^{\prime 2}, \alpha \geq 0$, under a pinching condition on the square of its mean curvature. Note that the hypothesis $(\log f)^{\prime \prime} \leq \alpha(\log f)^{\prime 2}$ is weaker than the widely used assumption $(\log f)^{\prime \prime} \leq 0$. In fact, in the framework of Mathematical Relativity, several energy conditions are usually assumed as the Timelike Convergence Condition (TCC), the Ubiquitous Energy Condition (UEC) and the Null Convergence Condition (NCC), among others. It is well known (see [9]) that in a Lorentzian warped product $\bar{M}=I \times{ }_{f} F$ obeying the TCC, its warping function satisfies $f^{\prime \prime} \leq 0$ and consequently the condition $(\log f)^{\prime \prime} \leq 0$ also holds. When the Lorentzian warped product satisfies the UEC, it holds the stricter condition $f^{\prime \prime}<0$ and so $(\log f)^{\prime \prime}<0$ [9]. On the other hand, when the fiber of a Lorentzian warped product obeying the NCC is Ricci-flat, then also $(\log f)^{\prime \prime} \leq 0[4]$.

Theorem 1 Let $\bar{M}=I \times_{f} F$ be a spatially parabolic covered Lorentzian warped product whose warping function is not globally constant and satisfies that $(\log f)^{\prime \prime} \leq \alpha(\log f)^{\prime 2}$, for a certain real constant $\alpha \geq 0$. Let $\psi: M \rightarrow \bar{M}$ be a complete hypersurface with bounded hyperbolic angle and such that $\sup f(\tau)<\infty$ and $\inf f(\tau)>0$. If $H^{2} \leq \frac{f^{\prime 2}(\tau)}{f^{2}(\tau)} \cosh ^{2} \varphi$, then $M$ is a spacelike slice.

Proof: First, notice that from the hypotheses it follows that $(M, \widehat{g})$ is parabolic (see Section 2.1).

Given a positive real number $\sigma$, it is a straightforward computation to get from (7) that

$$
\begin{aligned}
\widehat{\Delta} f^{-\sigma}(\tau)= & -\sigma f^{-\sigma-1}\left\{-n f(\tau) f^{\prime}(\tau)^{2}-n H f^{\prime}(\tau) f(\tau)^{2} \cosh \varphi\right. \\
& \left.+f(\tau)^{3}\left[(\log f)^{\prime \prime}(\tau)-(n+\sigma-3) \frac{f^{\prime}(\tau)^{2}}{f(\tau)^{2}}\right]|\nabla \tau|^{2}\right\} .
\end{aligned}
$$

Hence, taking $\sigma \geq 3$ such that $\sigma-3=\alpha$, (8) can be rewritten as

$$
\begin{aligned}
\widehat{\Delta} f^{-\sigma}(\tau)= & -\sigma f^{-\sigma-1}\left\{-n f(\tau) f^{\prime}(\tau)^{2} \cosh ^{2} \varphi-n H f^{\prime}(\tau) f(\tau)^{2} \cosh \varphi\right. \\
& \left.+f(\tau)^{3}\left[(\log f)^{\prime \prime}(\tau)-\alpha \frac{f^{\prime}(\tau)^{2}}{f(\tau)^{2}}\right]|\nabla \tau|^{2}\right\},
\end{aligned}
$$

where we have used (3) and (4).

Observe that from the assumptions on the warping function $f$ and the pinching condition for $H^{2}$, it follows that $\widehat{\Delta} f^{-\sigma}(\tau)$ is non negative. Thus, as $f^{-\sigma}(\tau)$ is bounded from below, it follows from the parabolicity of $(M, \widehat{g})$ that $f^{-\sigma}(\tau)$ is constant (i.e. $f(\tau)$ is constant) and $H^{2}=\frac{f^{\prime 2}(\tau)}{f^{2}(\tau)} \cosh ^{2} \varphi$.

If $M$ is a spacelike slice, i.e. $\tau$ is constant, the proof finishes. Otherwise, $J=\operatorname{Im} \tau$ is a subinterval of $I$ and $f_{\left.\right|_{J}}$ is constant, whence $f^{\prime}(t)=0$ for all $t \in J$. Hence, $f^{\prime}(\tau)$ vanishes identically and so $M$ is maximal.

Therefore, from (6), $\tau$ is a harmonic function on the parabolic Riemannian manifold $(M, \widehat{g})$. Finally, since $f(\tau)$ is constant and we are assuming that $f$ is not globally constant, it can be easily reasoned that $\tau$ is bounded from below or from above, and consequently $\tau$ is constant on $M$ (i.e. $M$ is a slice). 
Remark 2 Several examples of non-constant spacelike graphs can be provided when some of the hypotheses in Theorem 1 are removed. To justify this, we refer the reader to [34] where some examples for the maximal case are shown. On the other hand, from a physical point of view, the boundedness of the warping function (on the spacelike hypersurface), can be interpreted as the hypersurface cannot approach to a physical singularity.

As a consequence of Theorem 1 we obtain (compare with [28, Cor. 4.5], which is formulated in a much more restrictive ambient of dimension $(2+1))$,

Corollary 3 Let $\bar{M}=I \times_{f} F$ be a spatially parabolic covered Lorentzian warped product whose warping function is not globally constant and satisfies that $(\log f)^{\prime \prime} \leq \alpha(\log f)^{\prime 2}$, for a certain real constant $\alpha \geq 0$. The only maximal complete hypersurfaces of $\bar{M}$ with bounded hyperbolic angle and such that $\sup f(\tau)<\infty$ and $\inf f(\tau)>0$ are the spacelike slices.

Remark 4 Observe that Theorem 1 and Corollary 3 remain true if we change the assumption " $f$ is not globally constant" by " $M$ is contained in a (possibly open) slab", namely $\tau$ is bounded from below or from above. It can also be expressed, in a more physical language, asking the spacelike hypersurface to be bounded away from $-\infty$ or from $+\infty$.

On the other hand, recall that a Lorentzian warped product is said to be proper if the warping function $f$ is non-locally constant, i.e. there is no open subinterval $J(\neq \emptyset)$ of $I$ such that $f_{\left.\right|_{J}}$ is constant. Hence, the assumption " $f$ is not globally constant" is satisfied by requiring the Lorentzian warped product to be proper.

Remark 5 Let $\mathbf{H}=H N$ be the mean curvature vector at a point $p \in M$ and $\mathbf{H}_{\text {slice }}=\frac{f^{\prime}(\tau(p))}{f(\tau(p))} \partial_{t}(p)$ the mean curvature vector at the same point $\psi(p) \in \bar{M}$ for the spacelike slice $t=\tau(p)$. Then the assumption $H^{2} \leq \frac{f^{\prime 2}(\tau)}{f^{2}(\tau)} \cosh ^{2} \varphi$ admits an equivalent form in natural geometric terms regarding these vectors as

$$
|\bar{g}(\mathbf{H}, \mathbf{H})| \leq\left|\bar{g}\left(\mathbf{H}, \mathbf{H}_{\text {slice }}\right)\right|
$$

On the other hand, note that the extrinsic assumption $H^{2} \leq \frac{f^{\prime 2}(\tau)}{f^{2}(\tau)} \cosh ^{2} \varphi$ is weaker that the natural comparison hypothesis $H^{2} \leq \frac{f^{\prime 2}(\tau)}{f^{2}(\tau)}$ commonly used in several works (see [30], [33], [35], [10], [3], [19], [26]). This last inequality can be geometrically interpreted as follows: the mean curvature of the spacelike hypersurface, at any point, is bounded, in absolute value, by the mean curvature of the spacelike slice at that point.

In the following result we are able to keep away from the assumption on the lower bound of the warping function $f$ in Theorem 1 by asking $(\log f)^{\prime \prime}$ to satisfy a functional inequality, which in particular is satisfied when $\log f$ is convex.

Theorem 6 Let $\bar{M}=I \times_{f} F$ be a spatially parabolic covered Lorentzian warped product whose warping function is not globally constant and satisfies that $(\log f)^{\prime \prime} \leq(n-2+\alpha f)(\log f)^{\prime 2}$, for a certain real constant $\alpha>0$. Let $\psi: M \rightarrow \bar{M}$ be a complete hypersurface with bounded hyperbolic angle and such that $\sup f(\tau)<\infty$. If $H^{2} \leq \frac{f^{\prime 2}(\tau)}{f^{2}(\tau) \cosh ^{2} \varphi}$, then $M$ is a spacelike slice. 
Proof: Again, observe that from the hypotheses it follows that $(M, \widehat{g})$ is parabolic.

Consider now the function $e^{-\sigma f(\tau)}, \sigma>0$, which is bounded from above. From (7), its $\widehat{g}_{-}$ Laplacian can be easily calculated to obtain

$$
\begin{aligned}
\widehat{\Delta} e^{-\sigma f(\tau)}= & -\sigma e^{-\sigma f(\tau)}\left\{-n f(\tau) f^{\prime}(\tau)^{2}-n H f^{\prime}(\tau) f(\tau)^{2} \bar{g}\left(N, \partial_{t}\right)\right. \\
& \left.+f(\tau)^{3}\left[(\log f)^{\prime \prime}(\tau)-(n-2+\sigma f)(\log f)^{\prime}(\tau)^{2}\right]\right\} .
\end{aligned}
$$

Taking $\sigma=\alpha$, then $\widehat{\Delta} e^{-\sigma f(\tau)} \geq 0$. The parabolicity of $(M, \widehat{g})$ implies that $f(\tau)$ must be constant. The proof ends by a reasoning analogous to the one in Theorem 1 .

Corollary 7 Let $\bar{M}=I \times_{f} F$ be a spatially parabolic covered Lorentzian warped product whose warping function is not globally constant and satisfies that $(\log f)^{\prime \prime} \leq(n-2+\alpha f)(\log f)^{\prime 2}$, for a certain real constant $\alpha>0$. The only maximal complete hypersurfaces of $\bar{M}$ with bounded hyperbolic angle and such that $\sup f(\tau)<\infty$ are the spacelike slices.

\subsection{The case of monotonic warping function}

Now, we focus on the particular case where the warping function $f$ is monotone. This assumption has a natural physical interpretation. In fact, if for each $p \in F$ we parametrize $I \times\{p\}$ by $\gamma_{p}(t)=(t, p)$, since $\partial_{t}$ is the velocity of each galaxy $\gamma_{p}$, they are its integral curves. In particular, the function $t$ is the common proper time of all the galaxies. By taking $t$ as a constant, we get the hypersurface

$$
M(t)=\{t\} \times F=\{(t, p): p \in F\} .
$$

Then, the distance between two galaxies $\gamma_{p}$ and $\gamma_{q}$ in $M(t)$ is $f(t) d(p, q)$, where $d$ is the Riemannian distance in the fiber $F$. In particular, when $f$ has positive (resp. negative) derivative, the spaces $M(t)$ are expanding (resp. contracting).

Under such assumption, a primitive $\mathcal{F}(t)$ of $f(t)$ will be a suitable distinguished function to deal with our problems.

Given a positive continuous function $f$ on an interval $I=(a, b),-\infty \leq a<b \leq+\infty$, we will write $f \in L^{1}(a)$ (resp. $\left.f \in L^{1}(b)\right)$ to mean that $\int_{a}^{c} f(s) d s<+\infty$ (resp. $\int_{c}^{b} f(s) d s<+\infty$ ) for some (and therefore for every) $c \in(a, b)$. We will write $f \in L^{1}(I)$ to express that $f \in L^{1}(a)$ and $f \in L^{1}(b)$.

Throughout this section we will assume that the warping function $f$ is defined on $I=(a, b)$, $-\infty \leq a<b \leq+\infty$. If $f: I \longrightarrow \mathbb{R}$ verifies that $f \in L^{1}(a)$ (resp. $\left.f \in L^{1}(b)\right)$ and we fix $c \in(a, b)$, we will consider the functions

$$
\mathcal{F}(t)=\int_{a}^{t} f(s) d s \quad\left(\text { resp. } \mathcal{G}(t)=\int_{t}^{b} f(s) d s\right), \quad t \in(a, b),
$$

and $\mathcal{F}(\tau):=\mathcal{F} \circ \tau(\operatorname{resp} . \mathcal{G}(\tau):=\mathcal{G} \circ \tau)$. From (6) we get that

$$
\widehat{\Delta} \mathcal{F}(\tau)=-f(\tau)^{2} f^{\prime}(\tau)\left[n \cosh ^{2} \varphi-2 \sinh ^{2} \varphi\right]-n H f(\tau)^{3} \bar{g}\left(N, \partial_{t}\right) .
$$

Then we have 
Theorem 8 Let $\bar{M}=I \times_{f} F$ be a spatially parabolic covered Lorentzian warped product whose warping function is non-decreasing (resp. non-increasing). Let $\psi: M \rightarrow \bar{M}$ be a complete hypersurface with bounded hyperbolic angle and such that $\sup f(\tau)<\infty$.

$$
\text { If } H \leq 0 \text { and } f \in \mathrm{L}^{1}(a) \text { (resp. } H \geq 0 \text { and } f \in \mathrm{L}^{1}(b) \text { ), then } M \text { is a maximal spacelike slice. }
$$

Proof: Let us assume that $f$ is non-decreasing and $H \leq 0$. Then, defining the positive function $\mathcal{F}(t)$ as in $(9)$ and using $(10)$, it follows that $\mathcal{F}(\tau)$ is superharmonic for the metric $\widehat{g}$. Therefore, since $(M, \widehat{g})$ is parabolic, $\mathcal{F}$ is constant and we conclude that $M$ is a spacelike slice. The proof finishes observing that $\widehat{\Delta} \mathcal{F}(\tau)=0$ and all its addends have same sign.

The other case is analogous, now working with the function $\mathcal{G}(t)$.

Remark 9 If $f$ is non-decreasing (resp. non-increasing), and $a \neq-\infty$ (resp. $b \neq \infty$ ), taking into account that $f$ is positive and continuous, we do not have to assume the integrability condition, since $\mathcal{F}(t)=\int_{a}^{t} f(s) d s \quad\left(\right.$ resp. $\left.\mathcal{G}(t)=\int_{t}^{b} f(s) d s\right)$ is automatically finite in this case.

In particular, no convergence integral condition is necessary when the interval $I$ is finite. This fact must be borne in mind throughout all this subsection.

As can be easily checked, $n \cosh ^{2} \varphi-2 \sinh ^{2} \varphi \geq n \cosh \varphi$ for $n \geq 4$. Using that, we can derive the following consequence from (10).

Theorem 10 Let $\bar{M}=I \times_{f} F^{n}, n \geq 4$, be a spatially parabolic covered Lorentzian warped product whose warping function is non-decreasing (resp. non-increasing) and $f \in \mathrm{L}^{1}(a)$ (resp. $\left.f \in \mathrm{L}^{1}(b)\right)$. Let $\psi: M \rightarrow \bar{M}$ be a complete hypersurface with bounded hyperbolic angle and such that $\sup f(\tau)<$ $\infty$.

If $H^{2} \leq \frac{f^{\prime}(\tau)^{2}}{f(\tau)^{2}}$, then $M$ is a spacelike slice.

Proof: It follows from a reasoning analogous to the one in Theorem 8, by checking that under the assumptions the Laplacian (10) is non-positive (resp. non-negative).

For arbitrary dimension $n$, we can state the following result which can be proved alike.

Theorem 11 Let $\bar{M}=I \times_{f} F$ be a spatially parabolic covered Lorentzian warped product whose warping function is non-decreasing (resp. non-increasing) and $f \in \mathrm{L}^{1}(a)$ (resp. $f \in \mathrm{L}^{1}(b)$ ). Let $\psi: M \rightarrow \bar{M}$ be a complete hypersurface with bounded hyperbolic angle and such that $\sup f(\tau)<\infty$.

If $H^{2} \leq \frac{f^{\prime}(\tau)^{2}}{f(\tau)^{2} \cosh ^{2} \varphi}$, then $M$ is a spacelike slice.

Again for $n \geq 4$, we have

Theorem 12 Let $\bar{M}=I \times_{f} F^{n}, n \geq 4$, be a spatially parabolic covered Lorentzian warped product whose warping function $f \in \mathrm{L}^{1}(I)$ does not attain any local minimum. Let $\psi: M \rightarrow \bar{M}$ be a complete hypersurface with bounded hyperbolic angle and such that $\sup f(\tau)<\infty$.

If $H^{2} \leq \frac{f^{\prime}(\tau)^{2}}{f(\tau)^{2}}$, then $M$ must be a spacelike slice. 
Proof: From Theorem 10, we only have to study the case when $f$ changes from being non-decreasing to non-increasing. Let $t_{0}$ be a point where it happens, and consider the bounded function

$$
\mathcal{F}(t)=\int_{t_{0}}^{t} f(s) d s .
$$

Observe that $\mathcal{F} \geq 0$ when $t \geq t_{0}$, and $\mathcal{F} \leq 0$ when $t \leq t_{0}$. Taking again $\mathcal{F}(\tau)$, from (10) we obtain that $\mathcal{F} \widehat{\Delta} \mathcal{F} \geq 0$. The proof ends making use of [29, Lemma 2.2].

Now, given $c \in(a, b)$ let us take

$$
\mathcal{H}(t)=\int_{c}^{t} f(s)^{-1} d s
$$

and $\mathcal{H}(\tau)=\mathcal{H} \circ \tau$, whose $\widehat{g}$-Laplacian is given by

$$
\widehat{\Delta} \mathcal{H}(\tau)=-f(\tau)^{-1}\left[n f(\tau) f^{\prime}(\tau)+n H \bar{g}\left(N, \partial_{t}\right)\right] .
$$

Then we have

Theorem 13 Let $\bar{M}=I \times_{f} F$ be a spatially parabolic covered Lorentzian warped product whose warping function is non-increasing (resp non-decreasing) and $f^{-1} \in \mathrm{L}^{1}(b)$ (resp. and $f^{-1} \in \mathrm{L}^{1}(a)$ ). Let $\psi: M \rightarrow \bar{M}$ be a complete hypersurface with bounded hyperbolic angle and such that $\sup f(\tau)<$ $\infty$.

If $H \geq 0$ (resp. $H \leq 0)$ or $H^{2} \leq \frac{f^{\prime 2}(\tau)}{f^{2}(\tau)} \cosh \varphi$, then $M$ is a spacelike slice. Furthermore, in the first case $M$ is maximal.

Finally, in the spirit of Theorem 11 we have

Theorem 14 Let $\bar{M}=I \times_{f} F$ be a spatially parabolic covered Lorentzian warped product whose warping function does not attain any local minimum and $f^{-1} \in \mathrm{L}^{1}(I)$. Let $\psi: M \rightarrow \bar{M}$ be a complete hypersurface with bounded hyperbolic angle and such that $\sup f(\tau)<\infty$.

$$
\text { If } H^{2} \leq \frac{f^{\prime 2}(\tau)}{f^{2}(\tau)} \cosh \varphi \text {, then } M \text { is a spacelike slice. }
$$

Proof: Reasoning as in the proof of Theorem 11, we have to check what happens when $f$ changes from being non-decreasing to being non-increasing. Let $t_{0}$ be a point where it occurs, and let us consider the function

$$
\mathcal{H}(t)=\int_{t_{0}}^{t} f(s)^{-1} d s .
$$

Using (11), we arrive to $\mathcal{H}(\tau) \widehat{\Delta} \mathcal{H}(\tau) \geq 0$. Then, using [29, Lemma 2.2] we get that $\mathcal{H}$ is constant and the proof finishes. 


\subsection{Lorentzian products}

Next, we focus on the special class of Lorentzian products, i.e. those whose warping function is constant. Note that, for such ambient spacetimes, the spacelike slices are maximal. Given a spacelike hypersurface $\psi: M \rightarrow \bar{M}$ in a Lorentzian product, the Laplacian of $\tau$ with respect to the induced metric $g$ becomes (see 5)

$$
\Delta \tau=-n H \bar{g}\left(N, \partial_{t}\right)
$$

Then we have

Theorem 15 Let $\bar{M}=I \times F$ be a spatially parabolic covered Lorentzian product and $\psi: M \rightarrow \bar{M}$ a complete hypersurface with bounded hyperbolic angle. If $\tau$ is bounded from below (resp. above) and the mean curvature of $M$ is non-negative (resp. non-positive), then $M$ is a (maximal) spacelike slice.

Proof: From the hypotheses it follows that $(M, g)$ is parabolic. If $H$ is non-negative, then $\Delta \tau \geq 0$ and, since $\tau$ is bounded from below, it must be constant. The other case follows analogously.

This result improves [5, Cor. 8], where the same conclusion is achieved under the additional assumption of non-negative Ricci curvature of the fiber $F$ (which actually means that the Lorentzian products satisfies the TCC).

We can also provide the following non-existence result:

Theorem 16 Let $\bar{M}=I \times F$ be a spatially parabolic covered Lorentzian product. Then $\bar{M}$ does not admit any complete hypersurface whose hyperbolic angle is bounded and its mean curvature satisfies $\inf |H|>0$.

Proof: Let us suppose that such a hypersurface exists. Note that, from the assumption on $H$, it follows that the hypersurface cannot be a spacelike slice.

Consider the positive function $e^{\sigma \tau}$, where $\sigma$ is a non-zero real number. From (12), it is easy to check that

$$
\Delta e^{\sigma \tau}=\sigma e^{\sigma \tau}\left\{\sigma|\nabla \tau|^{2}-\bar{g}\left(N, \partial_{t}\right) H\right\}
$$

First, let us assume that the hypersurface has positive mean curvature and choose

$$
\sigma=\frac{-\inf H}{\sup ^{2} \sinh ^{2} \varphi}
$$

which is well-defined because $\sinh ^{2} \varphi$ cannot vanish identically on $M$ (otherwise $M$ would be a spacelike slice). Then, $e^{\sigma \tau}$ is a positive superharmonic function on the parabolic manifold $(M, g)$ and consequently it must be constant, leading to $\tau$ to be constant which is a contradiction.

The case of negative mean curvature can be analogously reasoned, taking now $\sigma=\frac{\inf |H|}{\sup _{\sinh }^{2} \varphi}$.

As a consequence we have

Corollary 17 In a spatially parabolic covered Lorentzian product, the only complete CMC hypersurfaces whose hyperbolic angle is bounded are the maximal ones. 


\section{Calabi-Bernstein type Problems}

Let $\left(F, g_{F}\right)$ be a (non-compact) $n$-dimensional Riemannian manifold and $f: I \longrightarrow \mathbb{R}$ a positive smooth function. For each $u \in C^{\infty}(F)$ such that $u(F) \subseteq I$, we can consider its graph $\Sigma_{u}=$ $\{(u(p), p): p \in F\}$ in the Lorentzian warped product $\left(\bar{M}=I \times_{f} F, \bar{g}\right)$. The graph inherits from $\bar{M}$ a metric, represented on $F$ by

$$
g_{u}=-d u^{2}+f(u)^{2} g_{F} .
$$

This metric is Riemannian (i.e. positive definite) if and only if $u$ satisfies $|D u|<f(u)$ everywhere on $F$, where $D u$ denotes the gradient of $u$ in $\left(F, g_{F}\right)$ and $|D u|^{2}=g_{F}(D u, D u)$. Note that $\tau(u(p), p)=$ $u(p)$ for any $p \in F$, and so $\tau$ and $u$ may be naturally identified on $\Sigma_{u}$.

When $\Sigma_{u}$ is spacelike, the unitary normal vector field on $\Sigma_{u}$ satisfying $\bar{g}\left(N, \partial_{t}\right)<0$ is

$$
N=\frac{1}{f(u) \sqrt{f(u)^{2}-|D u|^{2}}}\left(f(u)^{2} \partial_{t}+D u\right) .
$$

Then the hyperbolic angle $\varphi$, at any point of $M$, between the unit timelike vectors $N$ and $\partial_{t}$, is given by

$$
\cosh \varphi=\frac{f(u)}{\sqrt{f(u)^{2}-|D u|^{2}}}
$$

and the corresponding mean curvature function is

$$
H(u)=\operatorname{div}\left(\frac{D u}{n f(u) \sqrt{f(u)^{2}-|D u|^{2}}}\right)+\frac{f^{\prime}(u)}{n \sqrt{f(u)^{2}-|D u|^{2}}}\left(n+\frac{|D u|^{2}}{f(u)^{2}}\right) .
$$

In this section, our aim is to derive non-parametric uniqueness results from the parametric ones provided in Section 3. To do that, we need the induced metric $g_{u}$ to be complete. Observe that, in general, the induced metric on a closed spacelike hypersurface in a complete Lorentzian manifold could be non-complete (see, for instance, [6]). In our setting, we can derive the completeness of $\Sigma_{u}$ as follows [5, Lema 17]

Lemma 18 Let $\bar{M}=I \times_{f} F$ be a Lorentzian warped product whose fiber is a (non-compact) complete Riemannian manifold. Consider a function $u \in C^{\infty}(F)$, with $\operatorname{Im}(u) \subseteq I$, such that the entire graph $\Sigma_{u}=\{(u(p), p): p \in F\} \subset \bar{M}$ endowed with the metric $g_{u}=-d u^{2}+f(u)^{2} g_{F}$ is spacelike. If the hyperbolic angle of $\Sigma_{u}$ is bounded and $\inf f(u)>0$, then the graph $\left(\Sigma_{u}, g_{\Sigma_{u}}\right)$ is complete, or equivalently the Riemannian surface $\left(F, g_{u}\right)$ is complete.

Since in this setting the projection onto $F$ is a diffeomorphism, it follows that any entire graph is parabolic in a spatially parabolic GRW spacetime (see [32, Section 5.2] for further details). This fact means that the simply-connection assumption can be avoided for the non-parametric scenario.

As a consequence of Lemma 18 and the previous parametric results, we can state the following Calabi-Bernstein type Theorems.

Theorem 19 Let $(F, g)$ be a parabolic Riemannian $n$-manifold, $I \subseteq \mathbb{R}$ an open interval in $\mathbb{R}$ and $f: I \longrightarrow \mathbb{R}^{+}$a non globally constant function satisfying that $(\log f)^{\prime \prime} \leq \alpha(\log f)^{\prime 2}$ for a certain 
$\alpha \geq 0$ and such that $\sup f<\infty$ and inf $f>0$. Then the only entire solutions $u \in C^{\infty}(F)$, with $\operatorname{Im}(u) \subseteq I$, to the system of non-linear differential inequalities

$$
\begin{gathered}
H(u)^{2} \leq \frac{f^{\prime 2}(u)}{f^{2}(u)-|D u|^{2}} \\
|D u|<\lambda f(u), \quad 0<\lambda<1,
\end{gathered}
$$

are the constant functions $u=u_{0}$ with $H=\frac{f^{\prime}\left(u_{0}\right)}{f\left(u_{0}\right)}$.

Proof: First observe that, from (13), the constraint condition (15) can be written as

$$
\cosh \varphi<\frac{1}{\sqrt{1-\lambda^{2}}}
$$

Hence, (15) holds if and only if $\Sigma_{u}$ has bounded hyperbolic angle. Moreover, (15) also implies that the metric $g_{u}$ is spacelike, and furthermore it is complete from Lemma 18.

On the other hand, (14) means that

$$
H(u)^{2} \leq \frac{f^{\prime 2}(u)}{f^{2}(u)} \cosh ^{2} \varphi .
$$

Finally, the thesis follows from Theorem 1.

Remark 20 Note that the restriction (16) makes $H(u)$ into a uniformly elliptic operator.

Alternatively, Theorem 19 can be stated in the following terms, extending and improving [30, Th. 4.9].

Theorem 21 Let $(F, g)$ be a parabolic Riemannian $n$-manifold, $I \subseteq \mathbb{R}$ an open interval in $\mathbb{R}$ and $f: I \longrightarrow \mathbb{R}^{+}$a non globally constant function satisfying that $(\log f)^{\prime \prime} \leq \alpha(\log f)^{\prime 2}$ for a certain $\alpha \geq 0$ and such that $\sup f<\infty$ and inf $f>0$. Then the only bounded entire solutions $u \in C^{\infty}(F)$, with $\operatorname{Im}(u) \subseteq I$, to the system of non-linear differential inequalities

$$
\begin{gathered}
H(u)^{2} \leq \frac{f^{\prime 2}(u)}{f^{2}(u)-|D u|^{2}} \\
|D u|<\lambda f(u), \quad 0<\lambda<1,
\end{gathered}
$$

are the constant functions $u=u_{0}$ with $H=\frac{f^{\prime}\left(u_{0}\right)}{f\left(u_{0}\right)}$.

In general, both types of statements are possible for every parametric result in Section 4 . However, to avoid being repetitive, we have opted for stating our Calabi-Bernstein results as in Theorem 21.

As a consequence of Theorem 21 (see also Corollary 3), we have the following result for maximal graphs (compare with [15, Th. 6.2 and 6.3] and [31, Cor. 5.3], all of them for the particular case of surfaces). 
Corollary 22 Let $(F, g)$ be a parabolic Riemannian $n$-manifold, $I \subseteq \mathbb{R}$ an open interval in $\mathbb{R}$ and $f: I \longrightarrow \mathbb{R}^{+}$a non globally constant function satisfying that $(\log f)^{\prime \prime} \leq \alpha(\log f)^{\prime 2}$ for a certain $\alpha \geq 0$ and such that $\sup f<\infty$ and inf $f>0$. Then the only bounded entire solutions $u \in C^{\infty}(F)$, with $\operatorname{Im}(u) \subseteq I$, to the equation

$$
\begin{gathered}
H(u)=0, \\
|D u|<\lambda f(u), \quad 0<\lambda<1,
\end{gathered}
$$

are the constant functions $u=u_{0}$ with $f^{\prime}\left(u_{0}\right)=0$.

Similar results to the ones in Theorems 19, 21 and Corollary 22 can be derived from Theorem 6 and Corollary 7. Observe that the additional assumption inf $f>0$ must be required to assure completeness only when non-bounded solutions are studied.

Next, we provide several Calabi-Bernstein type results under the assumption of monotonicity of the warping function.

Theorem 23 Let $(F, g)$ be a parabolic Riemannian $n$-manifold, $I=(a, b),-\infty \leq a<b \leq+\infty$, an open interval in $\mathbb{R}$ and $f: I \longrightarrow \mathbb{R}^{+}$a non-decreasing (resp. non-increasing) function such that $f \in \mathrm{L}^{1}(a)$ (resp. $f \in \mathrm{L}^{1}(b)$ ) and $\sup f<\infty$ and $\inf f>0$. Then the only entire solutions $u \in C^{\infty}(F)$, with $\operatorname{Im}(u) \subseteq I$, to the system of non-linear differential inequalities

$$
\begin{gathered}
H(u) \leq 0 \quad(\text { resp. } H(u) \geq 0) \\
|D u|<\lambda f(u), \quad 0<\lambda<1,
\end{gathered}
$$

are the constant functions $u=u_{0}$ with $f^{\prime}\left(u_{0}\right)=0$.

Proof: It follows from Theorem 8.

Note that when $I$ is finite the integral convergence conditions can be omitted since they hold immediately (see Remark 9), and the same occurs when we study bounded entire solution. Thus, as a consequence of Theorem 23 we have

Corollary 24 Let $(F, g)$ be a parabolic Riemannian $n$-manifold, $I=(a, b),-\infty \leq a<b \leq+\infty$, an open interval in $\mathbb{R}$ and $f: I \longrightarrow \mathbb{R}^{+}$a non-decreasing (resp. non-increasing) function such that $\sup f<\infty$ and inf $f>0$. Then the only bounded entire solutions $u \in C^{\infty}(F)$, with $\operatorname{Im}(u) \subseteq I$, to the system of non-linear differential inequalities

$$
\begin{gathered}
H(u) \leq 0 \quad(\text { resp. } H(u) \geq 0) \\
|D u|<\lambda f(u), \quad 0<\lambda<1,
\end{gathered}
$$

are the constant functions $u=u_{0}$ with $f^{\prime}\left(u_{0}\right)=0$.

Although by stating our results as in Corollary 24 instead as in Theorem 23 we are losing some generality, we have opted for enunciating them in that way as we have been doing throughout this section.

For $n \geq 4$, we get as a consequence of Theorem 10 
Theorem 25 Let $(F, g)$ be a parabolic Riemannian $(n \geq 4)$-manifold, $I \subseteq \mathbb{R}$ an open interval in $\mathbb{R}$ and $f: I \longrightarrow \mathbb{R}^{+}$a non-decreasing (resp. non-increasing) function such that $\sup f<\infty$ and $\inf f>0$. Then the only bounded entire solutions $u \in C^{\infty}(F)$, with $\operatorname{Im}(u) \subseteq I$, to the system of non-linear differential inequalities

$$
\begin{gathered}
H(u)^{2} \leq \frac{f^{\prime}(u)^{2}}{f(u)^{2}} \\
|D u|<\lambda f(u), \quad 0<\lambda<1,
\end{gathered}
$$

are the constant functions $u=u_{0}$ with $H=\frac{f^{\prime}\left(u_{0}\right)}{f\left(u_{0}\right)}$.

For arbitrary dimension $n$, as a consequence of Theorem 11 we can state

Theorem 26 Let $(F, g)$ be a parabolic Riemannian $n$-manifold, $I \subseteq \mathbb{R}$ an open interval in $\mathbb{R}$ and $f: I \longrightarrow \mathbb{R}^{+}$a non-decreasing (resp. non-increasing) function such that $\sup f<\infty$ and inf $f>0$. Then the only bounded entire solutions $u \in C^{\infty}(F)$, with $\operatorname{Im}(u) \subseteq I$, to the system of non-linear differential inequalities

$$
\begin{gathered}
H(u)^{2} \leq \frac{f^{\prime}(u)^{2}\left(f^{2}(u)-|D u|^{2}\right)}{f(u)^{4}} \\
|D u|<\lambda f(u), \quad 0<\lambda<1,
\end{gathered}
$$

are the constant functions $u=u_{0}$ with $H=\frac{f^{\prime}\left(u_{0}\right)}{f\left(u_{0}\right)}$.

Proof: Observe that (17) means that

$$
H(u)^{2} \leq \frac{f^{\prime 2}(u)}{f^{2}(u) \cosh ^{2} \varphi} .
$$

The results follows from Theorem 11 .

Also for $n \geq 4$ we have from Theorem 12

Theorem 27 Let $(F, g)$ be a parabolic Riemannian $(n \geq 4)$-manifold, $I \subseteq \mathbb{R}$ an open interval in $\mathbb{R}$ and $f: I \longrightarrow \mathbb{R}^{+}$a function which does not attain any local minimum and such that $\sup f<\infty$ and $\inf f>0$. Then the only bounded entire solutions $u \in C^{\infty}(F)$, with $\operatorname{Im}(u) \subseteq I$, to the system of non-linear differential inequalities

$$
\begin{gathered}
H(u)^{2} \leq \frac{f^{\prime}(u)^{2}}{f(u)^{2}} \\
|D u|<\lambda f(u), \quad 0<\lambda<1,
\end{gathered}
$$

are the constant functions $u=u_{0}$ with $H=\frac{f^{\prime}\left(u_{0}\right)}{f\left(u_{0}\right)}$.

Now, from Theorems 13 and 14 we have 
Theorem 28 Let $(F, g)$ be a parabolic Riemannian $n$-manifold, $I \subseteq \mathbb{R}$ an open interval in $\mathbb{R}$ and $f: I \longrightarrow \mathbb{R}^{+}$a non-decreasing (resp. non-increasing) function such that $\sup f<\infty$ and inf $f>0$. Then the only bounded entire solutions $u \in C^{\infty}(F)$, with $\operatorname{Im}(u) \subseteq I$, to the system of non-linear differential inequalities

$$
\begin{gathered}
H(u) \leq 0 \quad(\text { resp. } H(u) \geq 0) \\
|D u|<\lambda f(u), \quad 0<\lambda<1,
\end{gathered}
$$

are the constant functions $u=u_{0}$ with $f^{\prime}\left(u_{0}\right)=0$.

Theorem 29 Let $(F, g)$ be a parabolic Riemannian $n$-manifold, $I \subseteq \mathbb{R}$ an open interval in $\mathbb{R}$ and $f: I \longrightarrow \mathbb{R}^{+}$a non-decreasing (resp. non-increasing) function such that $\sup f<\infty$ and inf $f>0$. Then the only bounded entire solutions $u \in C^{\infty}(F)$, with $\operatorname{Im}(u) \subseteq I$, to the system of non-linear differential inequalities

$$
\begin{gathered}
H(u)^{2} \leq \frac{f^{\prime 2}(u)}{f^{2}(u)-|D u|^{2}} \\
|D u|<\lambda f(u), \quad 0<\lambda<1,
\end{gathered}
$$

are the constant functions $u=u_{0}$ with $H=\frac{f^{\prime}\left(u_{0}\right)}{f\left(u_{0}\right)}$.

Theorem 30 Let $(F, g)$ be a parabolic Riemannian $n$-manifold, $I \subseteq \mathbb{R}$ an open interval in $\mathbb{R}$ and $f: I \longrightarrow \mathbb{R}^{+}$a function which does not attain any local minimum and such that $\sup f<\infty$ and $\inf f>0$. Then the only bounded entire solutions $u \in C^{\infty}(F)$, with $\operatorname{Im}(u) \subseteq I$, to the system of non-linear differential inequalities

$$
\begin{gathered}
H(u)^{2} \leq \frac{f^{\prime 2}(u)}{f^{2}(u)-|D u|^{2}} \\
|D u|<\lambda f(u), \quad 0<\lambda<1,
\end{gathered}
$$

are the constant functions $u=u_{0}$ with $H=\frac{f^{\prime}\left(u_{0}\right)}{f\left(u_{0}\right)}$.

Next, we will provide some type Calabi-Bernstein results derived from Section 3.2. To begin, as a consequence of Theorem 15 we have

Theorem 31 Let $(F, g)$ be a parabolic Riemannian n-manifold. Then the only entire solutions $u \in C^{\infty}(F)$ to the system of non-linear differential inequalities

$$
\begin{aligned}
\operatorname{div}\left(\frac{D u}{\sqrt{1-|D u|^{2}}}\right) & \geq 0 \quad\left(\operatorname{resp\cdot div}\left(\frac{D u}{\sqrt{1-|D u|^{2}}}\right) \leq 0\right) \\
& |D u|<\lambda, \quad 0<\lambda<1,
\end{aligned}
$$

which are bounded from below (resp. above) are the constant functions $u=u_{0}$.

From Theorem 16 we derive the following non-existence result 
Theorem 32 Let $(F, g)$ be a parabolic Riemannian $n$-manifold. Then there is no entire solutions $u \in C^{\infty}(F)$ to the system of non-linear differential inequalities

$$
\begin{gathered}
\inf \left|\operatorname{div}\left(\frac{D u}{\sqrt{1-|D u|^{2}}}\right)\right|>0 \\
|D u|<\lambda, \quad 0<\lambda<1 .
\end{gathered}
$$

Finally, we have

Corollary 33 Let $(F, g)$ be a parabolic Riemannian $n$-manifold and $H_{0} \in \mathbb{R}^{*}$ a non-zero real constant. Then there is no entire solutions $u \in C^{\infty}(F)$ to the equation

$$
\begin{gathered}
\operatorname{div}\left(\frac{D u}{\sqrt{1-|D u|^{2}}}\right)=H_{0} \\
|D u|<\lambda, \quad 0<\lambda<1 .
\end{gathered}
$$

\section{Acknowledgments}

The first author is partially supported by the Spanish MICINN Grant with FEDER funds MTM201343970-P. The second author is partially supported by the Spanish MICINN Grant with FEDER funds MTM2013-47828-C2-1-P and by the Junta de Andalucía Regional Grant with FEDER funds P09-FQM-4496.

\section{References}

[1] A. Albujer and L.J. Alías, Parabolicity of maximal surfaces in Lorentzian product spaces, Math. Z. 267 (2011), 453-464.

[2] A.L. Albujer and L.J. Alías, Calabi-Bernstein results for maximal surfaces in Lorentzian product spaces, J. Geom. Phys. 59 (2009), 620-631.

[3] A.L. Albujer, F.E.C. Camargo and H.F. de Lima, Complete spacelike hypersurfaces with constant mean curvature in a Robertson-Walker spacetime, Math. Proc. Cambridge Philos. Soc. 151 (2011), 271-282.

[4] J.A. Aledo and R.M. Rubio, Scalar curvature of spacelike hypersurfaces and certain class of cosmological models for accelerated expanding universes, J. Geom. Phys. 104 (2016) 128-136.

[5] J.A. Aledo, R.M. Rubio and A. Romero, Constant mean curvature spacelike hypersurfaces in Lorentzian warped products and Calabi-Bernstein type problems, Nonlinear Anal. 106 (2014), 57-69.

[6] L.J. Alías and P. Mira, On the Calabi-Bernstein theorem for maximal hypersurfaces in the Lorentz-Minkowski space, Publicaciones de la RSME 4 (2003), 23-55. 
[7] L.J. Alías, A. Romero and M. Sánchez, Uniqueness of complete spacelike hypersurfaces of constant mean curvature in Generalized Robertson-Walker spacetimes, Gen. Relativity Gravitation 27 (1995), 71-84.

[8] L.J. Alías, A. Romero and M. Sánchez, Spacelike hypersurfaces of constant mean curvature and Calabi-Bernstein type problems, Tôhoku Math. J. 49 (1997), 337-345.

[9] L.J. Alías, A. Romero and M. Sánchez, Spacelike hypersurfaces of constant mean curvature in spacetimes with symmetries, Publicaciones de la RSME 1 (2000), 1-14.

[10] C.P. Aquino and H.F. de Lima, On the rigidity of constant mean curvature complete vertical graphs in warped products, Differ. Geom. Appl. 29 (2011), 590-596.

[11] R. Bartnik, Quasi-spherical metrics and prescribed scalar curvature, J. Differential Geometry, 37 (1993), 31-71.

[12] D. Brill, Maximal Surfaces in closed and open spacetimes, Proceedings on the first Marcel Grossmann meeting on General Relativity, North-Holland Pub. Comp., Amsterdam (1977), 193-206.

[13] D. Brill and F. Flaherty, Isolated maximal surfaces in spacetime. Comm. Math. Phys. 50 (1976), 157-165.

[14] E. Calabi, Examples of Bernstein problems for some nonlinear equations, Proc. Symp. Pure Math. 15 (1970), 223-230.

[15] M. Caballero, A. Romero and R.M. Rubio, Uniqueness of maximal surfaces in Generalized Robertson-Walker spacetimes and Calabi-Bernstein type problems, J. Geom. Phys. 60 (2010) 394-402.

[16] M. Caballero, A. Romero and R.M. Rubio, New Calabi-Bernstein results for some elliptic nonlinear equations, Anal. Appl. 11 (2013), 1350002, 13 pp.

[17] M. Caballero, A. Romero and R.M. Rubio, Constant mean curvature spacelike hypersurfaces in Lorentzian manifolds with a timelike gradient conformal vector field, Class. Quantum Grav. 28 (2011), 145009(1-14).

[18] S.Y. Cheng and S.T. Yau, Maximal space-like hypersurfaces in the Lorentz-Minkowski spaces, Ann. Math. 104 (1976), 407-419.

[19] A.G. Colares and H.F. de Lima, Some rigidity theorems in Semi-Riemannian warped products, Kodai Math. J. 35 (2012), 268-282.

[20] I. Fernández and F.J. López, On the uniqueness of the helicoid and Eneper's surface in the Lorentz-Minkowski space $\mathbb{R}_{1}^{3}$. Trans. Amer. Math. Soc., 363 (2011), 4603-4650.

[21] F. Frankel, Applications of Duschek's formula to cosmology and minimal surfaces, Bull. Amer. Math. Soc. 81 (1975), 579-582.

[22] A. Grigoryan, Analytic and geometric background of recurrence and non-explosion of the Brownian motion on Riemannian manifolds, Bull. Amer. Math. Soc. 36(2) (1999), 135-249. 
[23] J. M. Latorre and A. Romero, New examples of Calabi-Bernstein problems for some nonlinear equations. Differ. Geom. Appl. 15 (2001), 153-163.

[24] G. Li and I Salavessa, Graphic Bernstein results in curved pseudo-Riemannian manifolds, J. Geom. Phys. 59 (2009), 1306-1313.

[25] A. Lichnerowicz, L'integration des equations de la gravitation relativiste et le probleme des $n$ corps, J. Math. Pures Appl. 23 (1944), 37-63.

[26] H.F. de Lima, On Bernstein-type properties of complete spacelike hypersurfaces immersed in a generalized Robertson-Walker spacetime, J. Geom. 103 (2012), 219-229.

[27] B. O’Neill, Semi-Riemannian Geometry with applications to Relativity, Academic Press, 1983.

[28] A. Romero and R.M. Rubio, On the mean curvature of spacelike surfaces in certain threedimensional Robertson-Walker spacetimes and Calabi-Bernstein type problems, Ann Glob Anal Geom 37 (2010), 21-31.

[29] A. Romero and R.M. Rubio, New proof of the Calabi-Bernstein theorem, Geom. Dedicata 147 (2010), 173-176.

[30] A. Romero and R.M. Rubio, A nonlinear inequality arising in geometry and Calabi-Bernstein type problems, J. Inequal. Appl. (2010), 950380, 10 pp.

[31] A. Romero and R.M. Rubio, On maximal surfaces in certain non-flat 3-dimensional RobertsonWalker Spacetimes, Math. Phys. Anal. Geom. 15 (2012), 193-202.

[32] A. Romero, R. Rubio and J.J. Salamanca, Uniqueness of complete maximal hypersurfaces in spatially parabolic generalized Robertson-Walker spacetimes, Class. Quantum Grav. 30 (2013), 115007, 13 pp.

[33] A. Romero, R. Rubio and J.J. Salamanca, Spacelike graphs of finite total curvature in certain 3-dimensional generalized Robertson-Walker spacetimes, Reports on Math. Phys. 73 (2014), 241-254.

[34] A. Romero, R. Rubio and J.J. Salamanca, A new approach for uniqueness of complete maximal hypersurfaces in spatially parabolic GRW spacetimes, J. Math. Anal. Appl., to appear.

[35] W. Wang and X. Liu, On a Bernstein type Theorems in Semi-Riemannian warped Products, Adv. Math. Phys. 2013, 959143, 5 pp.

[36] S.T. Yau, Some function-theoretic properties of complete Riemannian manifolds and their application to geometry, Indiana Univ. Math. J. 25 (1976), 659-670. 